\title{
Influence du détiquage sur la présence de parasites sanguins chez les bovins malgaches observés après splénectomie. Indications pratiques pour la lutte contre les hématozoaires pathogènes
}

\author{
par G. UILENBERG
}

\begin{abstract}
RÉSUMÉ
De nombreuses splénectomies ont montré que : le détiquage régulier, comme il eșt exéculé dans deux Centres de Recherches Zootechniques, empêche l'établissement de l'état de prémunition sur la plupart des bovins contre les babésioses et l'anaplasmose. Les bovins non détıqués deviennent tous infectés par Bobesia bigemina et Anaplasmo margmale ; B. argentina n'est pas toujours révélée par la splénectomie, et la proportion de bovins infectés est inconnue. Les résultats des splénectomies indiquent qu'A. marginale est habituellement transmis par la tique Baophlus microplus, mais les résultats des expériences de transmission sont en contradiction avec ces observations. Theilerio mutons ef les Eperythrozoon ne semblent pas être transmis habituellement par les tiques. Les résultats des splénectomies indıquent que B. microplus est peut-être le vecteur de l'hématozoarre Haematoxenus veliferus, mais la preuve expérimentale reste à faire. Les résultats obtenus fournissent la base pour la lutte contre les hématozoaıres palhogènes (Babesice et $A$. marginale). Il est nécessaıre de prémunir artıficiellement tout au moins les animaux cédés par les Centres.
\end{abstract}

Le détiquage est un des moyens classiques de lutte contre les maladies bovines causées par certains hématozoaires. Lorsqu'on utilsse le détiquage dans ce but, l'idéal à atteindre est d'élıminer toute tique dans le troupeau, ou même dans une régıon entière. Cet idéal est rarement réalisé, fait dô à plusieurs facteurs : contrôle insuffisant des animaux, manque d'efficacité du détıquage (résıstance des tiques à l'ixodicıde, concentration insuffisante, mauvaise méthode d'application, remanence insuffisante), apport de tiques des environs, etc.
Afin de mieux comprendre certains aspects de l'épizootologie des infections par les parasites sanguins à Madagascar, des recherches ont été entreprises pour déterminer l'influence d'un détiquage régulıer sur la présence de parasites sanguins, détiquage exécuté dans des carconstances pratıques, sans que les tıques soient entièrement élimınées.

La proportion des bovins détiqués montrant les différents parasites après splénectomie, est comparée à celle trouvée sur des onimaux non détıqués (RAYNAUD, 1962 (8) a déjà donné les 
résultats de splénectomies sur bovins détiqués ef non détiqués; le but de ses recherches n'était pas le même que le nôtre, et ses chiffres, cités plus loin, ne permettent pas pour la plupart des parasites sanguins de farre une comparaison entre les deux catégories d'anımaux.)

II ne sera question que des espèces dont la parasıtémie est augmentée par la splénectomie. Les espèces de ce groupe, trouvées à Madagascar, sont :

Babesia bigemina (SMITH et KILBORNE, 1893).

B. argentina (LIGNIĖRES, 1909).

Anoplasma marginale THEILER, 1910.

Theileria mutans (THEILER, 1906),

Eperythrozoon wenyon ADLER etELLENBOGEN, 1934.

E. teganodes HOYTE, 1962.

Hoematoxenus veliferus UILENBERG, 1964.

(Nous ne parlerons pas de l'espèce Anaplosma centrale THEILER, 1911, récemment importée et répandue par la prémunition contre l'anaplasmose.)

Les tiques des animaux domestiques, établies à Madagascar et trouvées sur les bovins, sont :

Boophilus microplus (CANESTRINI, 1887).

Amblyomma variegatum (FABRICIUS, 1794).

Otobius megnin (DUGĖS, 1883).

O. megnini et $A$. varlegatum ne transmettent pas, à notre connaissance, des parasites sanguins.

\section{MATÉRIEL ET MÉTHODES}

La technique opératorre pour les splénectomies est celle décrite par RAYNAUD (1961) (7), par résectıon de la $12^{\mathrm{e}}$ côte gauche, méthode qu permet un accès aisé à la rate et à son pédıcule. La méthode a seulement l'inconvénient que l'on ouvre la cavité thoracique dans une très grande proportion des cas ; l'on suture alors l'ouverture dans le diaphragme et nous n'en avons jamais vu de suites néfastes.

II a été possıble de splénectomiser en 1964, sur place, des bovins du Centre de Recherches Zootechniques de Miadana, alnsi que des animaux non détiqués des environs de ce Centre.

Le Centre est situé dans le nord-ovest de Madagascar, à environ $60 \mathrm{~km}$ de la côte, dans la régıon de Majunga. Les bovins y sont détıqués par douchage. L'on utilisait jusqu'au mois de septembre 1962, I'H. C. H. à une concentration de 0,02 p. 100 d'isomère gamma. L'H. C. H. a alors été remplacé par la Solution Bovine de COOPER (à base d'arsenite de sodium et d'acide crésylique), appliquée à une concentration de 1 p. 300 (solt 0,084 p. 100 d' $^{\prime} \mathrm{As}_{2} \mathrm{O}_{3}+0,046$ p. 100 d'acide crésylique). (Le changement de produit étalt nécessité par une épizootie de streptothrıcose cutanée). L'on douche les animaux hebdomadairement en saison des pluies (quand es tiques sont plus nombreuses), tous les 15 jours en saison sèche. Le détiquage à l'H. C. H. n'était pas entièrement efficace; un certain nombre de B. microplus (y compris des femelles gorgées) étaient présents, en 1962, sur les animaux du Centre. L'effet de l'arsenic semble meilleur ; Il n'y avaut, en 1964, que de très rares B. micropius (parmi lesquels iln'y avait qucune femellegorgée), en nombre beaucoup plus fable qu'en 1962. (Ce résultat est étonnant, puisque des expériences in vitro et in vivo ont montré que I'H.C. H. d̀ 0,02 p. 100 est beaucoup plus actif sur cette tique que le COOPER à 1 p. 300 . Il est possible qu'i y ait eu un début de résistance de $B$. microplus à I'H.C. H.). Outre les B. microplus, I'on trouve de faibles nombres d'A. variegatum. $O$. megnini ne semble pas exister dans la région.

Les bovins des environs du Centre, non détiqués, portent à longueur d'année de nombreux B. microplus et A. variegatum.

\section{REMARQUES}

La race des animaux n'est pas indiquée, l'expérience nous ayant montré qu'il n'existe aucune différence entre les races bovines quant à la réceptivité aux parasites sanguins. Notons. uniquement que tous les bovins splénectomisés du Centre et des environs étarent des mé-is Brahman - zébu local, avec des degrés différents de sang Brahmán.

B. bigemina, A. marginale et Th. mutans sont, d'après notre expérience, toujours décelés par la splénectomie, B. bigemina et Th. mutans en moins de 2 semaines, $A$. marginale en moins d'un mois. (RAYNAUD, 1962 (9), indique un maximum de 9 jours pour B. bigemina, de 34 jours pour A. marginale, mals ne mentionne pas le maximum pour Th. mutans). Les tables indiquent donc la totalité des porteurs de ces trois hématozocires. 
L'infection à $B$. argentına n'est certainement pas toujours révélée par la splénectomie; la rechute ne cause pas toujours de symptôme morbide et les parasites peuvent être si rares dans le sang que l'on ne les trouve pas; I'on peut déceler un plus grand nombre de porteurs en inoculant leur sang à des anımaux splénectomisés Indemnes, et en les injectant eux-mêmes avec du sang infectieux, procédure qui n'a pu être employée à Miadana. D'autre part, un cas a été observé où la rechute à $B$. argentina se faisait seulement 17 jours après l'opération (bien que RAYNAUD, 1962 (9), indique un maximum de 7 jours).

Le temps d'observation n'a donc peut-être pas été suffisant pour tous les animaux. Les chiffres n'indiquent vraisemblablement pas la totalité des porteurs de B. argentina.

Le temps d'observation nécessaire pour déceler avec certitude toutes les infections à $E$. teganodes et à $E$. wenyonı n'est pas connu ; d'autre part, les accès d'Eperythrozoon sont souvent fugaces et limités à de rares parasites, qui peuvent échapper à l'attention (UILENBERG (14)), Les chiffres $n$ 'indiquent donc peut-être pas la totalité des porteurs de ces organismes.

H. veliferus n'a pas encore été étudié de façon suffisamment approfondie pour que nous puissions être certain d'avoir décelé tous les porteurs.

\section{RÉSULTATS}

(Voir tableau No 1)

En résumé : B. bigemina 4 sur 16 ,

B. argentino 1 sur 16 ,

A. marginale 3 sur 16 ,

Th. mutons 15 sur 16 ,

E. wenyoni 15 sur 16 ,

E. tegonodes 3 sur 16 .

H. veliferus 9 sur 16 .

(Voir tableau no 2).

En résumé : $B$. bigemina 11 sur 11 ,

B. argentina 3 sur 11 ,

A. morginale 11 sur 11 ,

Th. mutons 11 sur 11 ,

E. wenyoni 11 sur 11 ,

E. teganodes 5 sur 11 ,

H. veliferus 11 sur 11 .

\section{DISCUSSION}

II ressort de ces résultats qu'un détıquage régulıer a une grande influence sur la présence de certains parasites sanguins, peu ou pas d'influence sur d'autres. Les résultałs permettent également de constater si certains parasites sont habituellement transmis par les tiques ou non.

Envisageons d'abord les hématozoaires pathogènes, $B$. bigemino, $B$. argentına et $A$. marginale. Les autres espèces semblent peu ou pas pathogènes : $T$. mutans peut causer une anémı, tout au moins sur les splénectomisés; les Eperythrozoon donnent lieu à de la fièvre et de l'anémıe, même sur les animaux intacts ; mais des mortalités dues à ces parasites n'ont pas été observées à Madagascar. H. veliferus n'a, jusqu'ici, pas montré de pouvoir pathogène.

Il ne fait aucun doute que la tique $B$. microplus est, à Madagascar, le vecteur habituel des deux espèces de Babesia; la fransmission expérimentale a été réussie au laboratoire à plusieurs reprises par notre prédécesseur RAYNAUD et par nous-mêmes.

Les chiffres dans les deux tables confirment que B. bigemina est habituellement transmise par les tıques, la différence entre les deux groupes étant statistiquement significative (Les limites de confiance $(P=0,05)$ sont respectivement de 7,3 à 51,2 p. 100 et de 71,7 à 100 p. 100 ). On peut également en conclure que le détiquage régulier, même sı de rares tiques sont présentes, empêche l'établissement de l'état de prémunition sur la plupart des animaux. Ces résultats sont d'ailleurs confirmés par d'autres splénectomies, qui n'ont pas été rapportées dans les tables. Elles ont été effectuées au Laboratoire de Tananarive, sur des veaux du C. R. Z. de Kıanjosoa.

Ce centre est situé à $180 \mathrm{~km}$ à l'ovest de Tananarive. La situation au point de vue tiques y est comparable à celle de Miadona : les bovins des environs, non détiqués, portent à longueur d'année de grands nombres de B. microplus et d'A. variegatum : $O$. megninı semble absent de la région. Les anımaux du Centre sont détiqués au bain, suivant le même protocole qu'à Miadana, maıs à de plus fortes concentrations de la Solutıon Bovine de COOPER à base d'arsenic : 1 p. 150 en saison sèche, 1 p. 200 en saıson des pluies.

B. microplus est normalement absent du Centre, 
TABIEAU NN I

Animaux du C.R.Z. de Miadana, régulièrement détıqués, spléneotomısés sur place en 1964

\begin{tabular}{|c|c|c|c|c|c|c|c|c|c|}
\hline \multirow{2}{*}{$\mathrm{N}^{\circ}$} & \multirow{2}{*}{ Age } & \multirow{2}{*}{ Observation } & \multicolumn{7}{|c|}{ Parasites observés } \\
\hline & & & B. bigemina & B. argentina & A. marginale & Th. mutans & E. wenyoni & E. tegenodes & H. veliferus \\
\hline $105 t$ & 10 mois & 36 jours & & & & & + & & \\
\hline 941 & $21 \quad 1$ & $50 "$ & & & & + & + & & + \\
\hline 918 & 2211 & $40 \quad 1$ & & & & + & + & & \\
\hline 912 & $22 \quad 11$ & $43 \quad 1$ & & & & + & & & \\
\hline 906 & $22 \quad 1$ & 3711 & & $\mathbf{T}$ & & + & + & & + \\
\hline 902 & $22 "$ & $48 \quad$ & & & & + & + & & \\
\hline 899 & $23 \quad 1$ & $371 "$ & + & & & + & + & + & \\
\hline 892 & 231 & 3711 & + & & & + & + & & + \\
\hline 877 & $23 \quad 1$ & $37 "$ & + & & & + & + & + & + \\
\hline 865 & $23 \quad 1$ & $37 \pi$ & & & & + & + & + & \\
\hline 859 & $24 \quad 1$ & $15 \pi$ & & & + & + & + & & + \\
\hline 856 & $24 \pi$ & 371 & & & & + & + & & \\
\hline 853 & $24 \pi$ & $36 "$ & + & & & + & + & & + \\
\hline 846 & $24 \pi$ & $37 "$ & & & & + & + & & + \\
\hline 663 & 4311 & $32 "$ & & & + & + & + & & + \\
\hline 518 & 6011 & 2311 & & & + & + & + & & + \\
\hline
\end{tabular}

(ND' = nứrro de l'animal; Observation = durée de l'examen quotidien du anng après l'opération)

TABLEAU N ${ }^{\circ}$ II

Animaux des environs de Miadana, non détiqués, splénectomisés sur place en 1964

\begin{tabular}{|c|c|c|c|c|c|c|c|c|c|c|c|}
\hline \multirow{2}{*}{$\mathrm{N}^{0}$} & \multirow{2}{*}{\multicolumn{2}{|c|}{ Age }} & \multirow{2}{*}{\multicolumn{2}{|c|}{ Observation }} & \multicolumn{7}{|c|}{ Parasiies observés } \\
\hline & & & & & B. bigemine & B. argentina & A. marginale & Th. motans & E. wenyonit & E. teganodes & H.veliferus \\
\hline 849 & 10 & moita & & ours & + & & + & + & + & & + \\
\hline 894 & 17 & $"$ & & $"$ & + & & + & + & + & & + \\
\hline 888 & 18 & $"$ & & "t & + & + & + & + & + & & + \\
\hline 885 & 18 & $"$ & & $"$ & + & & + & + & + & $\div$ & + \\
\hline 880 & 18 & $"$ & & $"$ & + & + & + & + & + & & + \\
\hline 871 & 19 & $"$ & & & + & & + & + & + & & + \\
\hline 820 & 22 & $"$ & & $"$ & + & & + & + & + & + & + \\
\hline 754 & 23 & $"$ & & $"$ & + & & + & + & + & + & + \\
\hline 752 & 23 & $"$ & & & + & & + & + & $1+$ & + & + \\
\hline 749 & 34 & " & & $"$ & + & & + & + & + & & + \\
\hline 694 & 36 & $"$ & & & + & + & + & + & + & + & + \\
\hline
\end{tabular}


A. variegotum y existe en faible nombre. Il y a de temps en temps introduction accidentelle de tiques avec des bovins de l'extérieur s'égarant dans le Centre; les larves à jeun de B. microplus qui en résultent, peuvent avoir l'occasion de se fixer temporairement sur les anımaux du Centre entre deux séances de détiquage (l'arsenic manquant de rémanence); $A$. variegatum est également introduit de cette façon, mais il y a, de plus, un apport sur des animaux sauvages (oiseaux, petits mammifères, etc...), sur lesquels I'on peut fréquemment trouver des larves et nymphes de cette espèce.

30 veaux du C. R. Z. de Kianjasoa, âgés de 4 à 9 mois, ont été splénectomısés en 1962-1964. 4 sur 30 seulement montraient $B$. bigemina. (Rappelons que RAYNAUD, 1962 (8), n'a trouvé aucun porteur sur 16 veaux de ce Centre, âgés de 3 à 12 mois.)

Ajoutons qu'aucun des 7 veaux du C.R. Z. de Miadana, âgés de 1 à 2 mols, n'a montré de B. bigemina après la splénectomie (1963-1964).

Nous n'avons pas de témoins non détiqués pour ces 37 vecux, strictement comparables au point de vue âge et régıon. Rappelons toutefois que RAYNAUD, 1962 (8), a trouvé 6 veaux sur 11 non détıquéz, âgés seulement de 2 d̀ 4 moıs, déjà porteurs de $B$. bigemina; ces veaux étaient originarres de la région de Tananarıve, où abondent les tiques $B$. microplus ef $O$. megnini, tandis qu'A. varregatum y est très rare. RAYNAUD, 1962 (8) et RAYNAUD et UILENBERG, 1962 (10) rapportent les résultats obtenus après splénectomie de 18 bovins adultes, non ou ırrégulièrement détıqués, de diverses régıons de Madagascar; tous étaient porteurs de B. bigemina.

II a été possible de splénectomiser encore, en 1965, 2 vaches adultes de la régıon de Tananarive; toutes les deux se révélaient être infectées.

\section{B. argentina :}

On ne peut tirer de conclusion nette de nos tables, le nombre d'animaux positifs après splénectomie étant faible sur les deux catégories de bovins. Signalons toutefols qu'aucun des 30 vequx du C. R. Z. de Kıanjasoa (4 à 9 mols), splénectomisés en 1962-1964, n'a montré ce parasite; il a été vérifié, par des inaculations de sang infecté, que 14 d'entre eux étaient en effet indemnes de $B$. argentina : ils présentalent après l'inoculation une crise parasitaire et thermıque. (Sur 16 veaux de 3 à 12 mois de ce Centre, RAYNAUD, 1962 (8), en trouve un seul positif.) RAYNAUD (8) signale 3 porteurs parmi les 11 veaux ( 2 à 4 mois) non détıqués de la région tananarivienne, mais aucun des 18 adultes (RAYNAUD, 1962 (8) et RAYNAUD et UILENBERG, 1962 (10) ne montrent le parasite; une des deux vaches splénectomisées en 1965, se révélalt être porteur. RAYNAUD ef UILENBERG, 1962 (10), pensent que ce parasite est un hématozoaire d'importation récente, encore limité à quelques régıons seulement du poys. UILENBERG, 1964 (13) signale que $B$. orgentino est plus répandue que l'on ne croyait auparavant Nous pouvons ajouter que toutes les souches de B. microplus, récoltées dans diverses régions du pays, mises sur bovins indemnes de Babesia, se sont révélées infectées de $B$. argentina, mals non toujours de $B$. bigemina. II est probable que $B$. argentina est également très répandue, mais qu'elle n'est souvent pas décelée par la splénectomie. D'allleurs s'il en était autrement, l'on devralt observer de nombreux cas de babésıellose sur des bovins non détiqués dans les régions où le parasite a été diagnostiqué ; or, il n'en est rien.

\section{A. morginale :}

La différence entre les deux groupes est statistiquement significative (Les limites de confiance $(P=0,05)$ sont respectivement de 4,1 à 44,7 p. 100 et de 71.7 à 100 p. 100.) Les résultats obtenus à Miadana indiquent danc que ce parasite est lui aussi habituellement transmis par les tiques, à l'opposé de ce que nous croyions auparavant (UILENBERG, 1964 (13)).

Cette première opinion était basée sur des résultats obtenus par le Dr. RAYNAUD, eł nos résultats préliminaıres sur des vecux de Kianjasoa, mals qui ne se sont pas confirmés par la suite. Le détiquage régulier empêche, comme pour $B$. bigemina, l'établissement de l'état de prémunition sur la plupart des animaux. Ceci est confirmé par d'autres splénectomies; aucun porteur sur 23 veaux du C. R. Z. de Kianjasoa, âgés de 4 à 9 mois; aucun porteur sur 5 veaux du C.R.Z. de Miadana, âgés de 1 à 2 mols. Par contre, 9 anımaux sur 11 non détıqués de la régıon de Tananarive, ôgés seulement de 2 à 4 mols (RAYNAUD, 1962 (8)), 19 bóvins adultes sur 19 de diverses régıons de Madagascar, non ou irrégulièrement détiqués (RAYNAUD, 1962 (8), 
RAYNAUD et UILENBERG, 1962 (10) et les 2 vaches splénectomisées en 1965), se sont montrés posıtifs. (Nous n'avons consıdéré que les anımaux qui ont pu être contrôlés pendant au moins un mols, ou qui se montraient positifs avant un mois.)

Il semble donc bien que la tique $B$. microplus soit le principal vecteur à Madagascar. (A. variegatum est très rare dans la région tananarivienne, où A. marginale est pourtant très fréquent: O. megnini semble absent de la plupart des régions où $A$. marginale est répandu ; les insectes piqueurs ailés ne sont pas influencés par le détiquage à l'arsenic dans les centres, où A. marglnale est pourtant rare.)

Cecl ne constitue évidemment qu'une forte présomption basée sur des observations. Les résultats expérimentaux obtenus à Madagascar, sont jusqu'ici en contradiction avec ces falts. RAYNAUD (Rapport Annuel de.1961 (5)) n'a pas réussi à transmettre $A$. marginale avec $B$. microplus ( 6 veaux splénectomisés observés pendant 40 d̀ 60 jours). Il est possible que la période d'observation alt été insuffisante, puisque THEILER, 1912 (12) et HENNING, 1956 (3) indiquent que la pérıde d'incubation après transmission par des tiques peut aller jusqu'à 100 jours ef plus.

Nous avons mis à intervalles réguliers des larves de B. microplus (de 50.000 à 100.000 larves environ par mois) sur un veau splénectomisé, indemne de tout parasite sanguin: les tique: provenaient de bovins du Laborataire, porteurs d'A. marginale (parfois nombreux dans le sang) et de bovins de l'extérieur, de diverses régıons du pays; jusqu'ici, 9 mols après le début de l'expérience, il n'y a pas eu apparition d'anaplasmes dans le sang du veau. (Le premier lot de larves transmettalt $B$. argentina, le cinquième lot $B$. bigemina; aucun autre parasite n'est apparu.) BRUMPT (1920) (1) n'a pas non plus réussi à transmettre A. marginale avec Margoropus australis ( $=$ B. microplus).

Le prablème de la transmission de l'anaplasmose à Madagascar mérite d'être étudié de façon approfondıe.

La discussion sur Th. mutans, les Eperythrozoo et $H$. veliferus sera limitée aux résultats obtenus en 1964 à Miadana.

\section{Th. mutans :}

II est évıdent que le détıquage n'a aucune influence sur la proportion de bovins infectés.
Cela confirme les expériences de CALLOW et HOYTE, 1961 (2), qui n'onf pas réussi d̀ transmettre Th. mutans avec B. microplus, tout comme RAYNAUD (Rapport Annuel, 1961 (5)), et nous-mêmes (le vecu mentıonné ci-dessus, sous A. marginale, auquel B. microplus n'a transmis jusqu'ici que les Babesiae). BRUMPT (1920) (1) ne réussit pas non plus la transmission avec une autre espèce de Boophilus (Margoropus calcoratus $=$ B. annulatus). Le vecteur habıtuel à Madagascar reste donc inconnu, mais il semble bien qu'il ne s'agısse pas d'une tique. II convient d'ajouter que les animaux nés et élevés à notre étable antı-tiques ne deviennent pas infectés spontanément, ce qui indıquerait qu'।l s'agit d'un vecteur qui est élıminé par les mesures de protection (entre autres : les animaux sont douchés deux fols par semaine à 0,425 p. 100 de carbaryl, produit actif contre certains insectes allés, par exemple les Hippoboscae, ainsı qu'll a pu être expérimenté récemment).

\section{Eperyfhrozoon :}

Il ne semble pas, d'après nos résultats, que les tiques soient les vecteurs, tout ou morns les plus importants, ainsı qu'וl a du reste déjà été démontré (UILENBERG (14)). Le vecteur habituel reste inconnu.

\section{H. veliferus :}

Les différences entre anımaux détiqués et non détiqués (respectivement 9 sur 16 et 11 sur 11) ne sont pas assez grandes pour conclure que les tiques soient les vecteurs. Toutefois, comme i) a été indiqué auparavant (UILENBERG (15), un examen détaillé des chiffres indique cue B. microplus pourrait être le vecteur. En divisant les animaux détiqués du Centre en deux groupes, un groupe dont les animaux ont apparemment été en contact avec B. microplus, puisqu'ils portent soit des Bobesiae, soit A. marginale, et un groupe dans lequel il n'y a pas de porteurs de ces hématozoarres, nous arrivons aux chiffres suivants :

I : Porteurs de Babesıa ou A. marginale : 7 sur 8 sont porteurs d'H. veliferus.

II : Animaux indemnes de Babesia et d'A. marginale: seulement 2 sur 8 montrent $H$. veliferus.

Les différences entre ces deux groupes ne sont 
toutefois pas statistiquement signıficatıves, les nombres d'anımaux étant trop faibles.

B. micropius serait-il un vecteur? Rappelons qu'H. veliferus n'a pas, jusqu'ic été transmis par B. microplus au veau mentionné ci-dessus (sous $A$. marginale), bien qu'une partie des tiques provient d'animaux ayant montré $H$. veliferus dans le sang.

\section{CONCLUSIONS}

Le détiquage régulier, comme il est exécuté dans deux Centres de Recherches Zootechniques à Madagascar, empêche l'établissement de l'état de prémunition contre les hématozoaıres pathogènes (Babesiae et $A$. marginale) sur la plupart des animaux. Les animaux non détiqués deviennent tous prémunis, tout au moins de manière certaine contre $B$. bigemına ef $A$. marginale.

Th. mutons et les Eperythrozoon ne semblent pas être transmis habituellement par les tiques; la transmission naturelle d' $H$. veliferus est encore incertaıne.

Les résultats des splénectomies peuvent être pris comme base de la lutte contre les hématozoaires pathogènes. Ils expliquent certains aspects de l'épizootologie de ces infections, à savoir que la quasi-totalité des cas cliniques de babésioses et d'anaplasmose sont observés à Madagascar sur les animaux régulièrement détiqués ; il est très rare de voir ces moladies sur des bovins non ou irrégulièrement détiqués. Les résultats de la présente enquête confirment les notions classiques dans ce domaine : le détiquage régulier, partiellement efficace, peut paradoxalement aboutir à un résultat opposé à celui recherché : il empêche l'établissement en bas âge de l'état de prémunition contre les hématozoaires pathogènes sur une proportion des anımaux: toutes les tiques n'étant pas éliminées, des cas de maladie grave, voire mortelle, peuvent se déclencher sur ces animaux plus tard, d̀ un âge ou ils sont devenus plus sensibles à ces affections. Il est également évident que les animaux cédés par les Centres aux éleveurs de l'extérieur deviendront rapidement malades dans une proportion importante par les babésıoses et par l'anaplasmose, puisque le détiquage à l'extérieur est rorement régulier et souvent absent; ils doivent souvent succomber lorsqu'il s'agit d'ani- maux adultes. Mais il n'a, jusqu'ıci, pas été possible de déterminer la part des pertes après cession qui incombe à chacune des infections transmises par les tiques (babésioses, anaplasmose, heartwater) et celle attribuable à d'autres maladies (dont la streptothricose cutanée est parmi les plus importantes).

Outre le traitement, souvent trop tardif lors de la forme cérébrale de la babésiellose ( $B$. orgentina) et de toute manière souvent inexistant en brousse, l'on peut envisager deux moyens de prévention :

a) Prémunition artificielle. II est nécessaire de prémunir tout au moins les bovins quittant les Centres, sous peine de fortes pertes après la cession.

La prémunition artificielle contre la piroplasmose vraie (B. bigemina) et l'anaplasmose ne semble plus poser de problème ; il a été possible de mettre au point une méthode sans danger pour la première maladie (Rapport Annuel, 1964) (6) ef la prémunition contre l'anaplasmose est facile et sans danger avec $A$. centrale. II n'a, par contre, pas encore été possible de mettre au point à Madagascar une méthode sûre et sans danger contre la babésiellose. Actuellement, les animaux allant quitter les Centres sont prémunis contre la piroplasmose vraie et l'anaplasmose et les recherches sur la bobésiellose sont poursuivies.

Les anımaux restant dans les Centres sont prémunis pendant leur première année contre l'anaplasmose (avec $A$. centrale), opération facile et qui assure l'état de prémunition pendant de nombreuses années, vraisemblablement toute la vie des anımaux ( $d$ 'après presque tous les auteurs, que nous n'énumérerons pas ici). II nous semble par contre superflu de prémunır les anımaux destinés à rester dans les Centres contre les babésioses tant que le détiquage régulier est contınué : l'état de prémunition est souvent perdu rapidement en l'absence de réinfections par les tiques (NEITZ, 1962 (4), RIEK, 1962 (11), UILENBERG, 1964 (13), et d'autres observations faites à Madagascar par la suite) ; en effet, l'état de prémunition contre $B$. bigemina est, dans notre expérience, en général perdu en moins d'un an, sans nouvelle infection. Ou alors, il faudrait refare cette prémunition artıficielle au moins une fois par an, opération coûteuse et 
rencontrant des difficultés d'exécution étant donné le grand nombre d'animaux dans les Centres.

b) Dımınuer le nombre de séances de détiquage afin d'arriver à un degré d'infestation par les tiques suffisant pour obtenir l'établissement ef l'entretien de l'état de prémunition sur tous les animaux dès leur jeunesse (lorsqu'ils sont beaucoup plus résistants à ces infections) ; l'infestation ne doit pas être assez importante pour nuire aux animaux par les effets directs (pertes de sang, portes d'entrée pour des bactéries pyogènes, etc...)

Cette méthode semble assez dangereuse puisque l'on ne connaît pas le degré d'infestation par les tiques nécessaire pour que tous les animaux soient prémunis et le restent ; seule l'expérence pourralt renseigner sur ce point. D'autre part, les animaux pourraient alors succomber à la heartwater (Cowdria ruminantium (COWDRY, 1925)), contre laquelle Madagascar ne dispose pas encore de vaccın. La streptothricose cutanée constitue également un grand problème ; elle est actuellement tenue en échec dans les Centres par l'application régulière de l'arsenic.

En résumé : le meilleur protocole de lutte contre les babésıoses et l'anaplasmose semble donc, dans les circonstances actuelles, se présenter comme suit :

Détiquer les animaux dans les Centres de la façon la plus efficace possible afin d'éviter les pertes par ces maladies (et par la heartwater et la streptothricose). Eviter, dans la mesure du possible, toute introduction accidentelle de B. microplus (empêcher que des bovins de l'extérieur s'y égarent).

Se contenter de traiter les quelques cas de babésıse qui se produiront dans les Centres malgré le détiquage, mais prémunir tous les jeunes animaux avec $A$. centrale.

Prémunir tous les animaux devant quitter les Centres contre la piroplasmose vraie ef l'anaplasmose et, lorsque cela sera possible, contre la babésiellose.

Ce protocole pourrait être modifié lorsque le laboratoire disposera d'un vaccin contre la heartwater ef d'un autre moyen que l'arsenic pour combattre la streptothricose ; I'on pourrait alors essayer une diminution du nombre de séances de détıquage afin d'arriver à la prémunıtion naturelle.

Nous remerçions MM. G. RASAONA et G. ANDRIANJAFY de leur collaboration technique.

\section{SUMMARY}

The influence of application of ixodicides on the presence of blood parasites in cattle in Madagascar, observed after splenectomy. Practical indications for control of pathogenic blood parasites

Numerous splenectomies have shown that:

- regular treatment with ixodicides, as carried out in two Centers for Zootechnical Research, prevents in the majority of cattle the establishment of the stale of premunity against babesiasis and anaplasmosis. Untreated cattle all become infected with Babesia bigemino and Anaplosma marginale : B argentina is not always detected by splenectomy, and the proportion of infected cattle is unknown. The results of the splenectomies indicate that $A$ morginale is habitually transmitted by the tick Boophilus microplus, but the results of transmission experiments are in contradiction with these observations Theileria mutans and the Eperythrozoon are apparently not habitually transmıtted by ticks. The results of the splenectomies indicate that $B$. microplus is perhaps the vector of the blood paraste Haematoxenus veliferus, but experimental proof has nol yet been obtained. These results furnish the base of control of the pathogenic blood parasites (Babesige and A marginale). Artificial premunising is necessary at least in animals that leave the Centers. 


\section{RESUMEN}

Influencia del desgarrapatazo sobre la presencia de parásitos sanguíneos en los bovinos malgachos observados después de una esplenectomia.

Indicaciones prácticas para la lucha contra los hematozoarios patógenos

Numerosas esplenectomias mostraron que :

- El desgarrapatazo regular, como el que se hace en los dos Centros de investıgaciones zootecnicos, impide el establecimiento del estado de premunición en la mayor parte de los bovinos contra las babesiosis y la anaplasmosis. Todos los bovinos no desgarrapatados se infectan con Babesia bigemina y Anaplasma marginale : no se descubre sımpre B. argentina mediante la esplenectomıa, y no se conoce la proporción de los bovinos infectados.

Los resultados de las esplenectomias indican que A marginale está transmitıda habitualmente por el ixodo Boaphilus microplus, pero los resultados de las experıencias de transmisión contradicen estas observacıones Theilerio mutans y los Eperythrozoon no parecen estar transmitidos habitualmente por los ixodos. Los resultados de las esplenectomias indican que $B$. microplus es acaso el vector del hematozoario Haematoxenus veliferus, pero hay que hacer la prueba experımental Los resultados obtenidos dan la base para la lucha contra los hematozoarios patógenos (Babesia y A. marginale). Es necesario precaver artificialmente a lo menos los animales dados por los Centros.

\section{BIBLIOGRAPHIE}

1. BRUMPT $\left(E_{1}\right)$. - Les piroplasmes des bovidés ef leurs hôtes vecteurs. Bull. Soc. Path. exot., 1920. $13: 416-460$.

2. CALLOW (L. L.) et HOYTE (H. M. D.). Transmission experiments using Babesia bigemina, Theileria mufans, Borrelia sp. and the cattle tick, Boophilus microplus. Aust. vet. J., 1961, $37: 381-390$.

3. HENNING (M. W.). - Animal diseases in South Africa. 3e édition. Central News Agency Ltd., South Africa, 1956.

4. NE!TZ (W. O.). - Rapport de la deuxième Réunion du Groupe d'Experts FAO/OIE sur les maladies du béfail transmises par des tiques. Le Caire, 1962. F. A. O. Rome.

5. Rapport annuel du laboratoire Central de l'Elevage de Tananarive, 1961.

6. - Rapport annuel du laboratoire Central de l'Elevage de Tananarive, 1964.

7. RAYNAUD (J. P.). - Une méthode de splénectomie des bovins adultes par résection de la $12^{\mathrm{e}}$ côte gauche. Rev. Elev. Méd. Vét. Poys trop., 1961, 14 : 321-327.

8. RAYNAUD (J. P.). - Prospection des hématozoaires et tiques de bovins à Madagascar. I. - Recherches dans la province de Tananarive. Rev. Elev. Méd. Vét. Pays trop., 1962, $15: 137-154$.

9. RAYNAUD (J. P.). - Splénectomie des bovins et parasites sanguins. Ann. Parasit. hum. comp., 1962, $37:$ 755-766.
10. RAYNAUD (J. P.) et UILENBERG (G.).-Prospection des hématozoaires et tiques de bovins à Madagascar. II. - Recherches complémentaires et conclusions. Rev. Elev. Méd. vét. Pays trop., 1962, 15 : 147-153.

11. RIEK (R. F.). - Rapport de la deuxième Réunion du Groupe d'Experts FAO/OIE sur les maladies du bétail transmises par des tiques. Le Caire, 1962. F. A. O., Rome.

12. THEILER (A.). - Gall-sickness of imported cattle and the protective inoculation against this disease. South Africon. Agriculfural Journal (janvier, 1912). Tiré-à-part no 6 , 1912 du Dept. of Agriculture, Union of South Africa.

13. UILENBERG (G.). -- Notes sur les hématozoaires ef tiques des animaux domestiques a Madagascar. (Avec une liste des Protozoaires, Rickettsiales et Arthropodes parasites, identifiés dans le pays.) Communication au Premier Congrès International de Parasitologie, Rome, septembre 1964. Rev. Elev. Méd. vét. pays trop., 1964,17, (3) : 337-359.

14. UILENBERG (G.). - Notes sur les Eperythrozoon de bovins à Madagascar. Rev. Elev. Méd. vét. pays trop., 1965, $18: 71-79$.

15. UILENBERG (G.). - Acquisitions nouvelles dans la connaissance d'Haematoxenus veliferus, hématozoaire des bovins à Madagascar. Bull. Soc. Path. exot. (sous-presse). 